\title{
Isomahanine induces endoplasmic reticulum stress and simultaneously triggers p38 MAPK-mediated apoptosis and autophagy in multidrug-resistant human oral squamous cell carcinoma cells
}

\author{
TANYARATH UTAIPAN ${ }^{1}$, ANAN ATHIPORNCHAI ${ }^{2,3}$, APICHART SUKSAMRARN $^{2}$, \\ SURASAK CHUNSRIVIROT ${ }^{4}$ and WARANGKANA CHUNGLOK ${ }^{1}$
}

\author{
${ }^{1}$ School of Allied Health Sciences, Walailak University, Nakhon Si Thammarat 80161; \\ ${ }^{2}$ Department of Chemistry and Center of Excellence for Innovation in Chemistry, Faculty of Science, \\ Ramkhamhaeng University, Bangkok 10240; ${ }^{3}$ Department of Chemistry and Center of Excellence \\ for Innovation in Chemistry, Faculty of Science, Burapha University, Chon Buri 20131; \\ ${ }^{4}$ Structural and Computational Biology Research Group and Department of Biochemistry, \\ Faculty of Science, Chulalongkorn University, Bangkok 10330, Thailand
}

Received July 11, 2016; Accepted December 5, 2016

DOI: $10.3892 / o r .2017 .5352$

\begin{abstract}
Advanced oral squamous cell carcinoma (OSCC) is typically aggressive and closely correlated with disease recurrence and poor survival. Multidrug resistance (MDR) is the most critical problem leading to therapeutic failure. Investigation of novel anticancer candidates targeting multidrug-resistant OSCC cells may provide a basis for developing effective strategies for OSCC treatment. In the present study, we investigated the cytotoxic mechanism of a carbazole alkaloid, namely isomahanine, in a multidrug-resistant OSCC cell line CLS-354/DX. We demonstrated that CLS-354/DX cells overexpressing multidrug resistance-associated protein 1 (MRP1) were resistant to anticancer drugs cisplatin and camptothecin. Isomahanine effectively induced cytotoxicity against CLS-354/DX cells regardless of resistance.
\end{abstract}

Correspondence to: Dr Warangkana Chunglok, School of Allied Health Sciences, Walailak University, 222 Thaiburi, Thasala, Nakhon Si Thammarat 80161, Thailand

E-mail: aonmu@hotmail.com; cwarang@wu.ac.th

Abbreviations: 3-MA, 3-methyladenine; 4-PBA, 4-phenylbutyric acid; BCRP, breast cancer resistance protein; $\mathrm{CHOP}$, transcription factor $\mathrm{C} / \mathrm{EBP}$ homologous protein; $\mathrm{CQ}$, chloroquine; ER, endoplasmic reticulum; LC3B, microtubule-associated protein light chain 3B; MAPK, mitogen-activated protein kinases; MDR, multidrug resistance; MRP, multidrug resistance-associated protein; OSCC, oral squamous cell carcinoma; p62, p62/SQSTM1; PARP, poly(ADP-ribose) polymerase; PERK, protein kinase PNA (PKR)-like ER kinase; P-gp, P-glycoprotein; UPR, unfolded protein response

Key words: isomahanine, oral squamous cell carcinoma, autophagy, apoptosis, multidrug resistance
Apoptosis as determined by FITC-Annexin V/PI staining and western blot analysis of cleaved caspase-3 and cleaved poly(ADP-ribose) polymerase (PARP) was significantly induced in a time-dependent manner upon isomahanine treatment. Isomahanine-induced caspase-dependent apoptosis was determined using z-VAD-fmk. The effects on autophagy in isomahanine-treated cells were investigated via conversion of LC3B and degradation of p62/SQSTM1 (p62). Isomahanine obviously induced autophagic flux as shown by an increase in punctate GFP-LC3B and the LC3B-II/LC3B-I ratio with a concomitant decrease in p62 levels. Autophagy inhibitors 3-methyladenine (3-MA) and chloroquine (CQ) protected isomahanine-induced cell death, indicating the activation of autophagic cell death. Endoplasmic reticulum (ER) stress and MAPK activation were examined to elucidate the mechanism underlying cell death. The expression levels of PERK, CHOP and phosphorylated MAPK (p38, ERK1/2 and JNK1/2) were upregulated following isomahanine treatment. We found that p38 MAPK inhibitor (SB203580) significantly attenuated isomahanine-induced apoptosis and autophagic flux and this prevented cell death. Collectively, the present study demonstrated that isomahanine was able to induce ER stress and trigger p38 MAPK-mediated apoptosis and autophagic cell death in multidrug-resistant OSCC cells. The potential cytotoxic action of isomahanine may provide the development of anticancer candidates for treating multidrug-resistant cancer.

\section{Introduction}

Oral squamous cell carcinoma (OSCC) is the most prevalent cancer occurring in the oral cavity and is the sixth most common cancer in Asia and the eleventh worldwide (1,2). The majority of OSCC patients are typically diagnosed when the cancer develops to an advanced stage, which confers a decrease of the 5-year survival rate to $20 \%$ (1). In this advanced-stage cancer, 
the phenomenon of multidrug resistance (MDR) is the most critical problem in chemotherapy, leading to therapeutic failure and disease recurrence (3). MDR can be attributed to multiple factors, such as upregulation of the ABC transporter family, aberrant apoptosis, change in DNA damage repair, miRNA regulation and cancer stem cell regulation (4). To circumvent the drug resistance of cancer cells, simultaneous activation of different cell-death pathways, such as apoptosis, autophagy and necroptosis, could be principally applicable (5-8). Hence, investigation of novel anticancer agents targeting contemporary non-apoptotic and apoptotic mechanisms may be a great step forward for multidrug-resistant OSCC treatment.

Apoptosis and autophagy are catalytic processes essential for maintaining cell populations and tissue homeostasis. Apoptosis can be initiated through mitochondrial-mediated and death receptor-mediated pathways. Both pathways lead to activation of a cascade of proteolytic caspases, thereby cleaving cytosolic and nuclear proteins, resulting in nuclear fragmentation and apoptotic death (9). Apoptosis is well-known programmed cell death type-1, which has been utilized as a cell-death mechanism to eliminate cancer cells by a number of anticancer compounds (10). Meanwhile, autophagy is a conserved catabolic process whereby damaged cellular constituents are sequestered into double-membrane vesicles known as autophagosomes, which are subsequently degraded by lysosomal machinery (11). During autophagy, the formation of autophagosomes is processed by a conversion of microtubuleassociated protein 1 light chain 3 (LC3-I to LC3-II). When the process progresses to the lysosomal degradation step, the cellular p62/SQSTM1 (p62) level is then degraded, and this whole process is referred to as autophagic flux (12). Autophagy plays a pro-death role or pro-survival role by being a 'doubleedged sword'. The pro-death role of autophagy occurs when autophagy is excessively activated, and this has shed light on its anticancer potential (13). The potential of these two cell death pathways to effectively eliminate multidrug-resistant cancer cells has been demonstrated $(5,6,14)$.

Various stress conditions can trigger these cell-death pathways. Endoplasmic reticulum (ER) stress with the consequent unfolded protein response (UPR) is one of the stress responses that confer apoptosis and autophagy (15). Components involved in ER stress and UPR signaling, such as inositol-requiring protein-1 and transcription factor C/EBP homologous protein (CHOP), modulate the mitogen-activated protein kinase (MAPK) signaling pathway, particularly p38 MAPK and JNK, which are important mediators promoting cell death $(16,17)$. Accumulating studies have demonstrated that activated p38 MAPK and JNK are involved in apoptosis and/or autophagy in various cancer cell lines (18-20).

Collective evidence has revealed that carbazole alkaloids exhibit a wide range of anticancer activities demonstrated both in vitro and in vivo (21-23), leading research in carbazole alkaloids to become more attractive in the pharmacological field in the last few decades (24). Isomahanine, a bioactive carbazole alkaloid abundantly found in Murraya koenigii, and its isomer mahanine are capable of inducing apoptosis in human leukemia cells via a caspase-dependent pathway (22). Relevant to multidrug-resistant cancers, mahanine can enhance the chemosensitivity of colon and cervical cancer cells to the anticancer drugs cisplatin and 5-fluorouracil $(25,26)$. Carbazole derivatives have been documented to upregulate autophagy and sensitize glioblastoma cells to anticancer drugs (27). However, the function of isomahanine in multidrug-resistant OSCC, along with the underlying mechanisms, have not yet been investigated.

In the present study, we aimed to investigate the cytotoxic mechanisms of isomahanine via apoptosis and autophagy in our established multidrug-resistant OSCC cell line (CLS-354/DX). We found that CLS-354/DX cells exhibited overexpression of multidrug resistance-associated protein 1 (MRP1) protein and a low responsiveness to conventional anticancer drugs. Isomahanine exerted an effective cytotoxic effect against CLS-354/DX cells regardless of their resistance compared to the parental cell line (CLS-354/WT). The compound induced ER stress and simultaneously triggered apoptosis and autophagic flux through the p38 MAPK signaling pathway. This finding supports the potential use of isomahanine to circumvent MDR in OSCC cells.

\section{Materials and methods}

Preparation of isomahanine. The bioactive carbazole alkaloid, i.e., isomahanine, was isolated and purified from the $\mathrm{CH}_{2} \mathrm{Cl}_{2}$ extract of $M$. koenigii leaves by repeated silica gel and Sephadex LH-20 column chromatography. The spectroscopic data of isomahanine were consistent with the reported values (28). The purified carbazole alkaloid was dissolved in dimethyl sulfoxide (DMSO) prior to conducting experiments.

Reagents and antibodies. cis-diamminedichloroplatinum(II) (cisplatin), camptothecin, DMSO, 3-MA, 4-phenylbutyric acid (4-PBA), chloroquine (CQ) and 3-(4,5-dimethylthiazol-2-yl)-2,5-diphenyltetrazolium bromide (MTT) were purchased from Sigma-Aldrich Corp. (St. Louis, MO, USA). Benzyloxycarbonyl-ValAla-Asp (OMe) and fluoromethylketone (z-VAD-fmk) were purchased from InvivoGen (San Diego, CA, USA). Luminita ${ }^{\mathrm{TM}}$ Chemiluminescent HRP substrate was purchased from EMD Millipore (Billerica, MA, USA). All primary antibodies (rabbit), HRP-conjugated secondary antibodies (anti-rabbit) and MAPK inhibitors (U0126, SB203580 and SP600125) were purchased from Cell Signaling Technology, Inc. (Danvers, MA, USA).

Cell lines and culture. Human OSCC CLS-354 cells (CLS Cell Lines Service GmbH, Eppelheim, Germany) at passage no. 30-40 were cultured in RPMI-1640 medium supplemented with $10 \%$ fetal bovine serum (Biochrom $\mathrm{GmbH}$, Berlin, Germany), $1 \%$ penicillin/streptomycin and $2 \mathrm{mM}$ stable L-glutamine (PAA Laboratories $\mathrm{GmbH}$, Pasching, Austria). The cancer cell line was maintained in an atmosphere of $95 \%$ humidity and $5 \% \mathrm{CO}_{2}$ at $37^{\circ} \mathrm{C}$. CLS-354 cells, as parental cells, were oral epithelial cancer cells exhibiting epithelial-like shape, namely CLS-354/WT (Fig. 1A, left). The mesenchymal phenotype with the elongated shape was isolated by differential trypsinization as previously described (29). The cell line was named as CLS-354/DX (Fig. 1A, right).

MDR assay. Cancer cells $\left(8 \times 10^{5}\right)$ were seeded onto a $60-\mathrm{mm}$ tissue culture dish and allowed to grow for $36 \mathrm{~h}$. Cells $\left(2 \times 10^{5}\right.$ cells/sample) were collected, washed with 
A

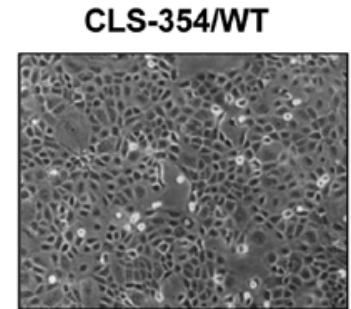

B

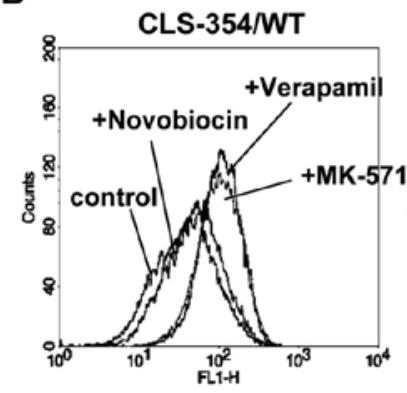

CLS-354/DX

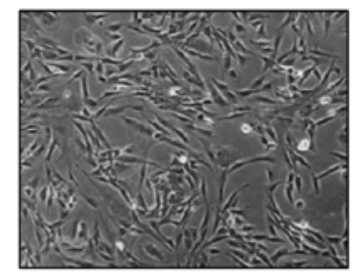

C

\begin{tabular}{|ccc|}
\hline Mean MAF & CLS-354/WT & CLS-354/DX \\
\hline P-gp & $61.66 \pm 1.09$ & $66.48 \pm 0.73 \S$ \\
MRP1/2 & $64.12 \pm 0.62$ & $86.10 \pm 0.58 \#$ \\
BCRP & $12.25 \pm 4.09$ & $16.55 \pm 4.21$ \\
\hline
\end{tabular}

D
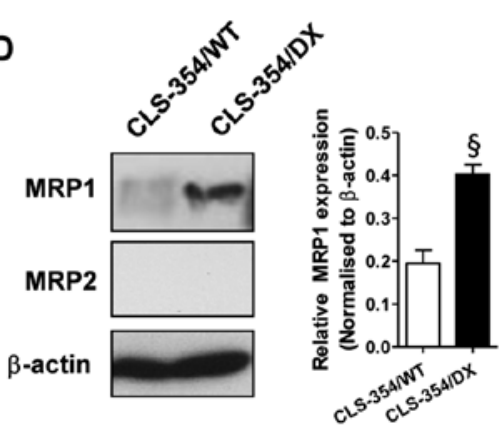

Figure 1. CLS-354/DX cells exhibit multidrug resistance (MDR) through MRP1 overexpression. (A) The cell morphology of the OSCC cell line CLS-354/WT and mesenchymal-like phenotype of CLS-354/DX were observed under a phase contrast microscope. (B) Cells were incubated with eFluxx-ID ${ }^{\circledR}$ Green probe in the absence or presence of inhibitors specific for P-gp (20 $\mu \mathrm{M}$ verapamil), MRP1/2 (50 $\mu \mathrm{M}$ MK-571) and breast cancer resistance protein (BCRP) (0.05 $\mu \mathrm{M}$ novobiocin). Then, cells were analyzed by flow cytometry. Histograms represent fluorescence intensity indicating the dye that accumulated in the absence or presence of the inhibitors. (C) MAF for each transporter indicates the quantitative characteristics of MDR. (D) The expression levels of proteins MRP1 and MRP2 were determined by western blotting (left), and the relative expression level of MRP2 protein was determined by densitometric analysis (right). Data are expressed as the mean \pm SEM $(\mathrm{n}=4) ;{ }^{\circledR} \mathrm{p}<0.01,{ }^{\#} \mathrm{p}<0.001$ vs. the CLS-354/WT cells.

phosphate-buffered saline (PBS), and subjected to MDR assay using eFluxx-ID ${ }^{\circledR}$ Green MDR assay kit (Enzo Life Sciences, Inc., Farmingdale, NY, USA) according to the manufacturer's instructions. The cells were analyzed immediately by flow cytometry. The mean fluorescence intensity (MFI) values were analyzed using CellQuest ${ }^{\mathrm{TM}}$ Pro (BD Biosciences, San Jose, CA, USA). The MDR activity factor (MAF) for each transporter was calculated according to the formula: $\mathrm{MAF}=100 \mathrm{x}\left(\mathrm{MFI}_{\mathrm{with} \text { inhibitor }}-\mathrm{MFI}\right.$ control $) / \mathrm{MFI}_{\text {with inhibitor }}$.

Cell viability assay. Cells $\left(1.5 \times 10^{4}\right.$ cells/well $)$ were seeded into 96-well plates and grown for $48 \mathrm{~h}$. Cells were treated with various concentrations of cisplatin (10-25 $\mu \mathrm{M})$ and camptothecin $(0.3-15 \mu \mathrm{M})$ or isomahanine $(10-25 \mu \mathrm{M})$ for $24 \mathrm{~h}$ or for various time-points. After treatment, cell viability was assessed by MTT assay. The half-maximal inhibitory concentration $\left(\mathrm{IC}_{50}\right)$ was calculated from concentrationresponse curves following a 24-h treatment. The resistance index (RI) was defined as the ratio of the $\mathrm{IC}_{50}$ value of the resistant cell line to the $\mathrm{IC}_{50}$ value of the parental cell line of each drug.

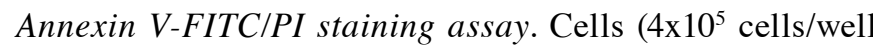
seeded on a 6 -well plate) were incubated with isomahanine for 0, 6, 12 and $24 \mathrm{~h}$. The cells were harvested, washed with PBS, and stained with Annexin V-FITC and Annexin V-FITC/PI according to the manufacturer's instructions (Roche Diagnostics Deutschland GmbH, Mannheim, Germany). Fluorescence intensity (FL-1 and FL-2) was immediately determined by flow cytometry. For each measurement, at least 15,000 cells were counted. The percentages of Annexin V-FITC- and Annexin V-FITC/PI-positive cells were analyzed as apoptotic cells using CellQuest ${ }^{\mathrm{TM}}$ Pro (BD Biosciences).
Analysis of GFP-LC3B puncta. CLS-354/DX cells $\left(2 \times 10^{4}\right.$ cells/well seeded on a 24 -well plate) were transduced with BacMam LC3B-GFP viral particles $(\mathrm{MIO}=30$ plaque-forming units/cell), according to the manufacturer's instructions of the Premo ${ }^{\mathrm{TM}}$ Autophagy Sensor kit (Invitrogen, Carlsbad, CA, USA). After $24 \mathrm{~h}$, the cells were treated with $20 \mu \mathrm{M}$ isomahanine or $50 \mu \mathrm{M}$ CQ. After a 16-h incubation, green fluorescent signals were monitored and imaged using a Zeiss Axio Vert.A1 inverted microscope (magnification, $x 40)$. Cells with >5 GFP-LC3B puncta were considered autophagy-positive. The percentage of cells with punctate GFP-LC3B/total GFP-LC3B-positive cells was determined.

Western blot analysis. After isomahanine treatment, the cells were harvested and lysed in lysis buffer containing protease inhibitors. Protein samples $(50 \mu \mathrm{g})$ were then prepared in Laemmli sample buffer. The proteins were separated on 8-12.5\% SDS-PAGE gels and transferred to a polyvinylidene fluoride (PVDF) membrane. The membrane was blocked with $5 \%$ non-fat dry milk, washed with a mixture of Tris-buffered saline and Tween-20, and probed with primary antibodies (1:250-1:1,000) at $4^{\circ} \mathrm{C}$ overnight. The blot was then probed with the HRP-conjugated secondary antibody $(1: 5,000)$ at room temperature for $1 \mathrm{~h}$. The proteins were visualized using the chemiluminescent detection system.

Statistical analysis. All results are expressed as the mean \pm SEM. Data were obtained from at least 3 independent experiments. Significant differences among the different groups were considered at a p-value $<0.05$, using Student's t-test or one-way ANOVA. The statistical analyses were performed using GraphPad Prism 6 (GraphPad Software, Inc., La Jolla, CA, USA). 
A

Cisplatin<smiles>N[PH](N)(Cl)Cl</smiles>

B

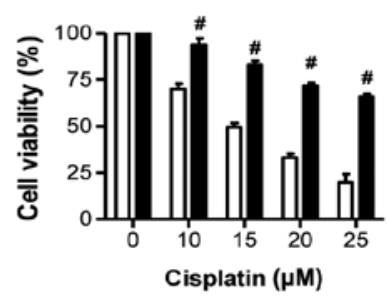

Camptothecin

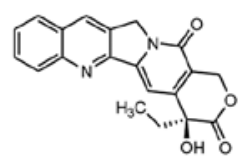

Isomahanine

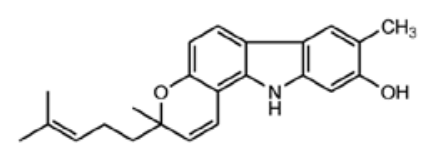

C

\begin{tabular}{|cccr|}
\hline IC $_{50}(\mu \mathrm{M})$ & CLS-354/WT & CLS-354/DX & RI \\
\hline Cisplatin & $14.67 \pm 0.44$ & $33.23 \pm 0.96$ & 2.27 \\
Camptothecin & $0.23 \pm 0.02$ & $>15.00$ & $>65.22$ \\
Isomahanine & $14.45 \pm 0.78$ & $16.00 \pm 0.65$ & 1.11 \\
\hline
\end{tabular}

Figure 2. Isomahanine exerts cytotoxicity against CLS-354/DX cells. (A) Chemical structures of cisplatin (left), camptothecin (middle) and isomahanine (right), (B) The cell viability of the CLS-354/WT and CLS-354/DX cell lines following treatment with cisplatin $(10-25 \mu \mathrm{M})$ (left), camptothecin $(0.3-2.5 \mu \mathrm{M})(\mathrm{middle})$ and isomahanine (10-25 $\mu \mathrm{M}$ ) (right) for $24 \mathrm{~h}$ was assessed by MTT assay. (C) The $\mathrm{IC}_{50}$ values of cisplatin, camptothecin and isomahanine in the CLS-354/WT and CLS-354/DX cell lines. The resistance index (RI) corresponded to the ratio of the $\mathrm{IC}_{50}$ value between the CLS-354/DX and CLS-354/WT cell lines. Data are expressed as the mean \pm SEM from at least 3 independent experiments; ${ }^{\circledR} \mathrm{p}<0.01,{ }^{\sharp} \mathrm{p}<0.001 \mathrm{vs}$. the CLS-354/WT cells.

\section{Results}

CLS-354 cells develop a multidrug-resistant phenotype. The multidrug-resistant OSCC cell line was spontaneously generated from the parental human OSCC cell line CLS-354 (passage no. 27), which is epithelial-like in shape (CLS-354/WT) (Fig. 1A, left). After being cultured for $>10$ passages, some cells became elongated and mesenchymal-like. These mesenchymal-like cells were isolated by differential adhesion (29) and named CLS-354/DX (Fig. 1A, right). These two cell lines had similar growth characteristics. The population doubling times of CLS-354/WT and CLS-354/DX were $31.25 \pm 0.64$ and $29.17 \pm 1.20 \mathrm{~h}$, respectively, which were not significantly different.

To investigate the drug-resistance profiling, the activity of a particular multidrug-resistant transporter [P-gp, MRP1/2 and breast cancer resistance protein (BCRP)] was assessed using eFluxx-ID ${ }^{\circledR}$ Green probe. The probe is a hydrophilic fluorescent dye, which is trapped within the cells unless actively pumped out by these transporters and is detectable by flow cytometry. As shown in Fig. 1B, CLS-354/WT cells exhibited an equal increase of fluorescent signals in the presence of a P-gp inhibitor (verapamil) and an MRP1/2 inhibitor (MK-571) in comparison to the control (Fig. 1B, left). We clearly found a higher fluorescent signal from the CLS-354/DX cells in the presence of verapamil and MK-571 when compared with the control (Fig. 1B, right). This result indicated the essential roles of P-gp and MRP1/2 in these cells. The comparative analysis of MDR between the CLS-354/WT and CLS-354/DX cell lines was estalished by calculating the MAF values. The MAF values of P-gp and MRP1/2 in CLS-354/DX cells were $66.48 \pm 0.73$ and $86.10 \pm 0.58$, respectively, which were significantly greater than those of the CLS-354/WT cells (Fig. 1C).
As a result, the highest MAF value of MRP1/2 was observed in the CLS-354/DX cells (Fig. 1C). Hence, the expression levels of the MRP1 and MRP2 proteins in these cell lines were examined by western blotting. We found that the MRP1 expression level in the CLS-354/DX cells was significantly greater than that in the CLS-354/WT cell line (Fig. 1D), while MRP2 was undetectable in both cell lines. These results indicated that the CLS-354/DX cells developed the multidrug-resistant phenotype via MRP1 overexpression.

Isomahanine has potential cytotoxicity against CLS-354/DX cells. The responsiveness to cisplatin, a platinum-based anticancer drug and camptothecin, an anticancer alkaloid (Fig. 2A, left and middle), was investigated in the CLS-354/WT and CLS-354/DX cells following 24-h treatments using the cell viability assay. The results showed that the response of CLS-354/DX cells to cisplatin and camptothecin was significantly lower than that of CLS-354/WT cells (Fig. 2B, left and middle). The $\mathrm{IC}_{50}$ values for CLS-354/DX cell line to cisplatin and camptothecin were $33.23 \pm 0.96$ and $>15 \mu \mathrm{M}$, respectively, which were 2 times and $>65$ times, respectively, greater than those of the CLS-354/WT cells as indicated by the RI (Fig. 2C). These data strongly support the contribution of MDR in CLS-354/DX cells. Notably, isomahanine (Fig. 2A, right) at a concentration starting from $20 \mu \mathrm{M}$ effectively suppressed the viability of CLS-354/DX cells without relevance of resistance (Fig. 2B, right). The $\mathrm{IC}_{50}$ values observed in both cell lines were not significantly different, with an RI value of 1.1 (Fig. 2C).

Isomahanine induces apoptosis in CLS-354/DX cells through a caspase-dependent pathway. The mechanism of cell death was further examined in the CLS-354/DX cells. Cell 
A

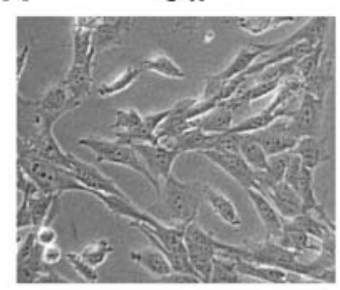

$12 \mathrm{~h}$

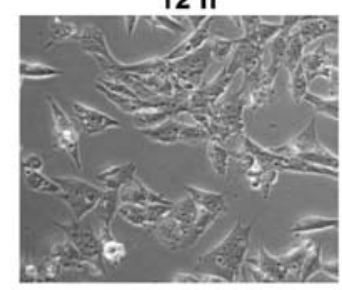

C

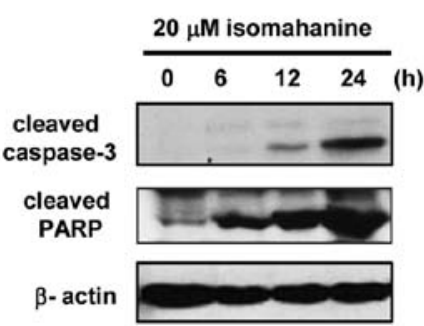

$6 \mathrm{~h}$

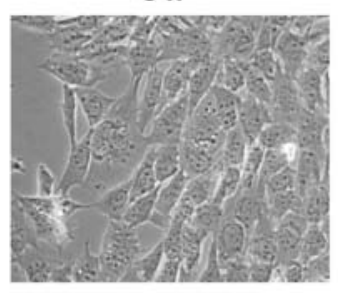

$24 \mathrm{~h}$

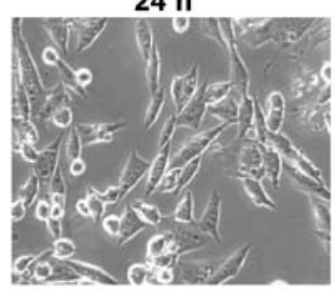

B
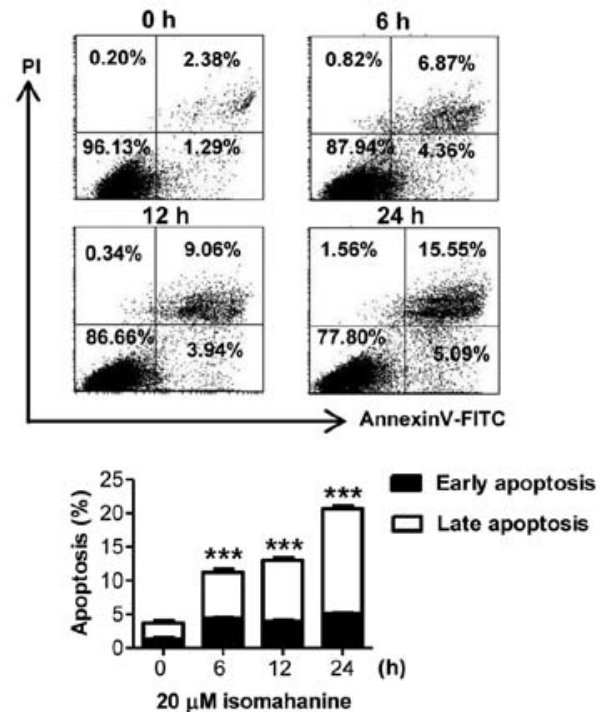

D
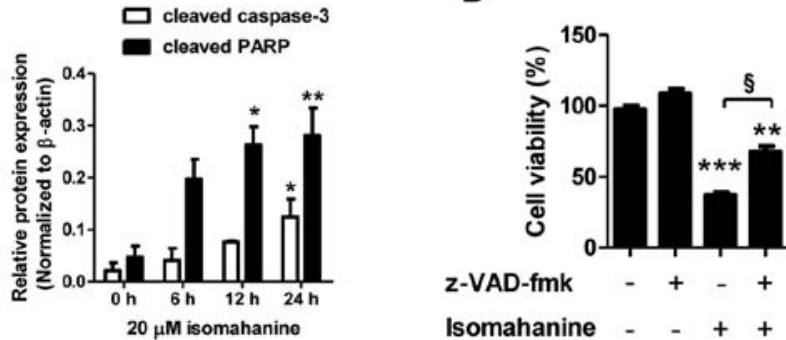

Figure 3. Isomahanine triggers apoptosis in CLS-354/DX cells. (A) The cell morphology of CLS-354/DX cells was monitored under a phase-contrast microscope (40X, objective) following treatment with isomahanine $(20 \mu \mathrm{M})$ for $0,6,12$, and $24 \mathrm{~h}$. (B) Apoptosis was assessed by Annexin V-FITC/PI double staining assay using flow cytometric analysis. (C) The expression levels of cleaved caspase-3 and cleaved PARP were determined by western blotting (left) and densitometric analyses of the relative expression level (right). (D) The cell viability of the CLS-354/DX cells following isomahanine (20 $\mu \mathrm{M})$ treatment in the absence or presence of z-VAD-fmk $(50 \mu \mathrm{M})$ was determined by MTT assay. Data are expressed as the mean \pm SEM of 3 independent experiments; ${ }^{*} p<0.05$, ${ }^{* *} \mathrm{p}<0.01,{ }^{* * *} \mathrm{p}<0.001$ vs. $0 \mathrm{~h} ;{ }^{s} \mathrm{p}<0.01$ vs. $\mathrm{z}-\mathrm{VAD}$-fmk co-treatment.

morphology was preliminarily observed in a time-course study following isomahanine treatment $(20 \mu \mathrm{M})$ for $0,6,12$ and $24 \mathrm{~h}$. As shown in Fig. 3A, the cells after exposure to isomahanine for $6 \mathrm{~h}$ presented small cytoplasmic vacuoles. After $12 \mathrm{~h}$, the cells detached and shrank in size. Eventually, several cells became round in shape and cell death followed after 24-h treatment. Hence, apoptosis was further confirmed by flow cytometric analysis as well as western blot analysis of caspase-3 and poly(ADP-ribose) polymerase (PARP). Isomahanine time-dependently induced early apoptotic and late apoptotic cells to 5.09 and $15.55 \%$, respectively (Fig. 3B, upper panel). Total apoptosis significantly increased in a time-dependent manner, while the maximal induction was increased to $20 \%$ following the 24-h treatment (Fig. 3B, lower panel). Isomahanine activated cleavage of caspase-3 and PARP in a time-dependent manner, and the greatest level of increase was observed upon 24-h treatment (Fig. 3C). To confirm caspase-dependent apoptotic death, the cells were treated with isomahanine along with $\mathrm{z}$-VAD-fmk, a pan-caspase inhibitor. The inhibition of caspase activity significantly rescued isomahanine-induced cell death (Fig. 3D). Thus, isomahanine induced apoptotic death via a caspase-dependent pathway.

Isomahanine induces autophagic flux in CLS-354/DX cells. We hypothesized that autophagy may be involved in cell death upon the morphological observations. We determined autophagic induction by analysis of GFP-LC3B puncta. Cells treated with isomahanine displayed a punctate pattern of GFP-LC3B localized in the perinuclear region, indicating autophagic induction. Isomahanine-induced GFP-LC3B puncta formation by $50 \%$, which was comparable to that of CQ $(\sim 60 \%)$, a positive control (Fig. 4A). We further performed western blotting of microtubule-associated protein 1B-light chain 3 (LC3B-II), which is an autophagosomal marker. Isomahanine induced the expression of LC3B-II in a time-dependent manner, and the expression of LC3B-II/LC3B-I ratio significantly increased after 12-h treatment (Fig. 4B). Thus, isomahanine induced autophagy in CLS-354/DX cells.

An increase in the number of autophagosomes may be due to activation or inhibition of autophagic flux. During autophagy, the cellular level of the p62 protein is typically degraded, and its decreased level serves as an indicator of autophagic flux (14). We further examined the expression of p62 with isomahanine treatment. Isomahanine markedly decreased the expression level of p62 in contrast with LC3B-II after $24 \mathrm{~h}$, indicating autophagic flux (Fig. 4C). We also excluded the effect of proteasomal degradation activity on the p62 protein using MG-132, a proteasome inhibitor. The result revealed that isomahanine decreased the level of p62 as usual, while MG-132 did not inhibit its decrease (Fig. 4D). To assess the contribution of autophagy to cell death, the effect of autophagy 

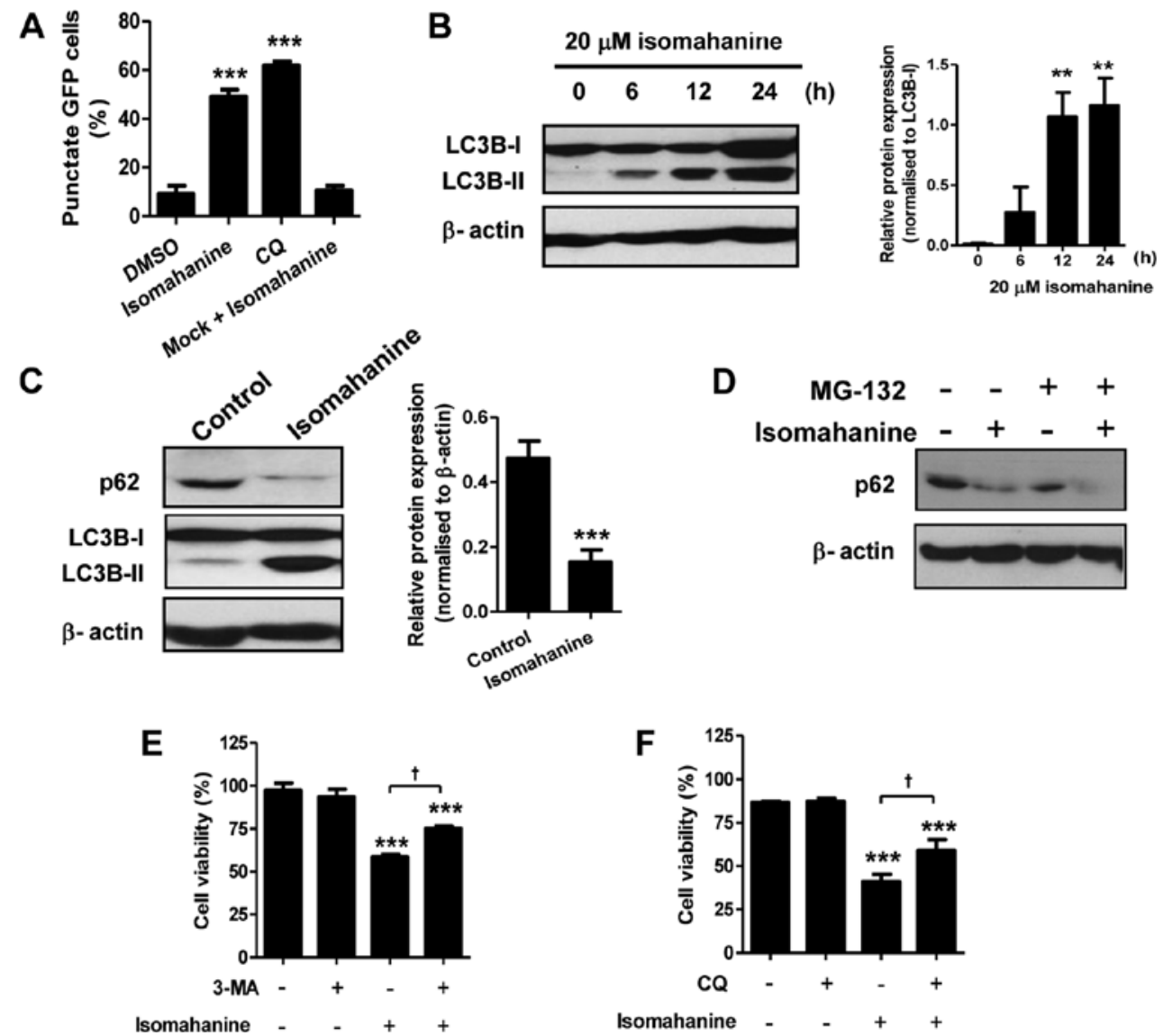

Figure 4. Isomahanine induces autophagic flux in CLS-354/DX cells. The cells were treated with isomahanine (20 $\mu \mathrm{M})$ for $0-24 \mathrm{~h}$. (A) GFP-LC3B expression was analyzed by transduction with BacMam LC3B-GFP or BacMam LC3B (G120A)-GFP (Mock). After treatment with isomahanine (20 $\mu$ M) or chloroquine $(50 \mu \mathrm{M})$ for $16 \mathrm{~h}$, a punctate pattern of GFP fluorescence was monitored under a phase contrast microscope (x40, magnification). Statistical analysis of the percentage of cells with GPF-LCB puncta was determined. Data are expressed as the mean \pm SEM of 4 independent experiments; ${ }^{* * *} \mathrm{p}<0.001 \mathrm{vs.}$ the DMSO control. (B) The expression of autophagosome marker (LC3B) was determined by western blotting (left) and densitometric analyses of the relative expression level (right). (C) The expression levels of autophagic flux markers (LC3B and p62) after treatment with isomahanine (20 $\mu \mathrm{M})$ for $24 \mathrm{~h}$ were determined by western blotting (left) and densitometric analyses of the relative expression level (right). (D) The expression level of p62 with isomahanine treatment in the absence or presence of MG-132 $(0.5 \mu \mathrm{M})$ at $24 \mathrm{~h}$ was determined by western blotting. The cell viability of the CLS-354/DX cells was assessed by MTT assay after a 24-h treatment with isomahanine $(20 \mu \mathrm{M})$ with or without (E) a 3-h pretreatment with 3-MA $(2.5 \mathrm{mM})$ or $(\mathrm{F})$ a co-treatment with CQ $(30 \mu \mathrm{M})$. Data are expressed as the mean \pm SEM of 3 independent experiments; ${ }^{* *} \mathrm{p}<0.01,{ }^{* * * *} \mathrm{p}<0.001 \mathrm{vs} .0 \mathrm{~h}$ or control without any treatment; ${ }^{\dagger} \mathrm{p}<0.05 \mathrm{vs}$. the isomahanine treatment alone.

inhibitors on cell viability was determined. Results revealed that the autophagy inhibitors 3-MA and CQ significantly inhibited isomahanine-induced cell death in the CLS-354/DX cells (Fig. 4E-F). Collectively, these findings suggest that autophagic flux induced by isomahanine was involved in the cytotoxic effects of the CLS-354/DX cells.

Isomahanine induces ER stress and MAPK activation in CLS-354/DX cells. To further elucidate the cell-death mechanisms induced by isomahanine, we examined the effect of this compound on the activation of ER stress and MAPK phosphorylation. As shown in Fig. 5A, isomahanine activated the phosphorylation of protein kinase PNA (PKR)-like ER kinase (PERK), an ER stress sensor, in a time-dependent manner. Concomitantly, the compound induced the expression of the transcription factor CHOP, which is a marker of UPR (Fig. 5A). Activation of the MAPK family members, p38, ERK1/2 and JNK1/2 was also determined. The phosphorylation of p38, ERK1/2 and JNK1/2 was markedly upregulated after exposure to isomahanine for $6 \mathrm{~h}$. At $12 \mathrm{~h}$ of treatment, the phosphorylation of p38 and ERK1/2 was strongly activated, while their activation slightly decreased after $24 \mathrm{~h}$ (Fig. 5A). JNK1/2 was activated to a maximal level at $6 \mathrm{~h}$ and decreased after $12 \mathrm{~h}$ (Fig. 5A). Next, we applied 4-PBA, an inhibitor of ER stress, to demonstrate the relation between ER stress and MAPK pathway. As shown in Fig. 5B, the activated PERK and $\mathrm{CHOP}$ proteins were significantly decreased in the presence of 4-PBA. ER stress inhibition protected against isomahanine-activated p38 MAPK phosphorylation. Meanwhile, the activation of ERK1/2 and JNK1/2 were concomitantly increased by 4-PBA (Fig. 5B). Collectively, these results suggest that ER stress mediated the activation of p38 MAPK, but not that of ERK1/2 and JNK1/2.

Isomahanine induces p38 MAPK-mediated apoptosis and autophagy in CLS-354/DX cells. Activation of MAPK in response to ER stress can promote cell death by apoptosis and autophagy. To study the role of MAPK in apoptosis- and autophagy-induced cell death, we assessed the cell viability with isomahanine treatment in the presence of inhibitors of 
A

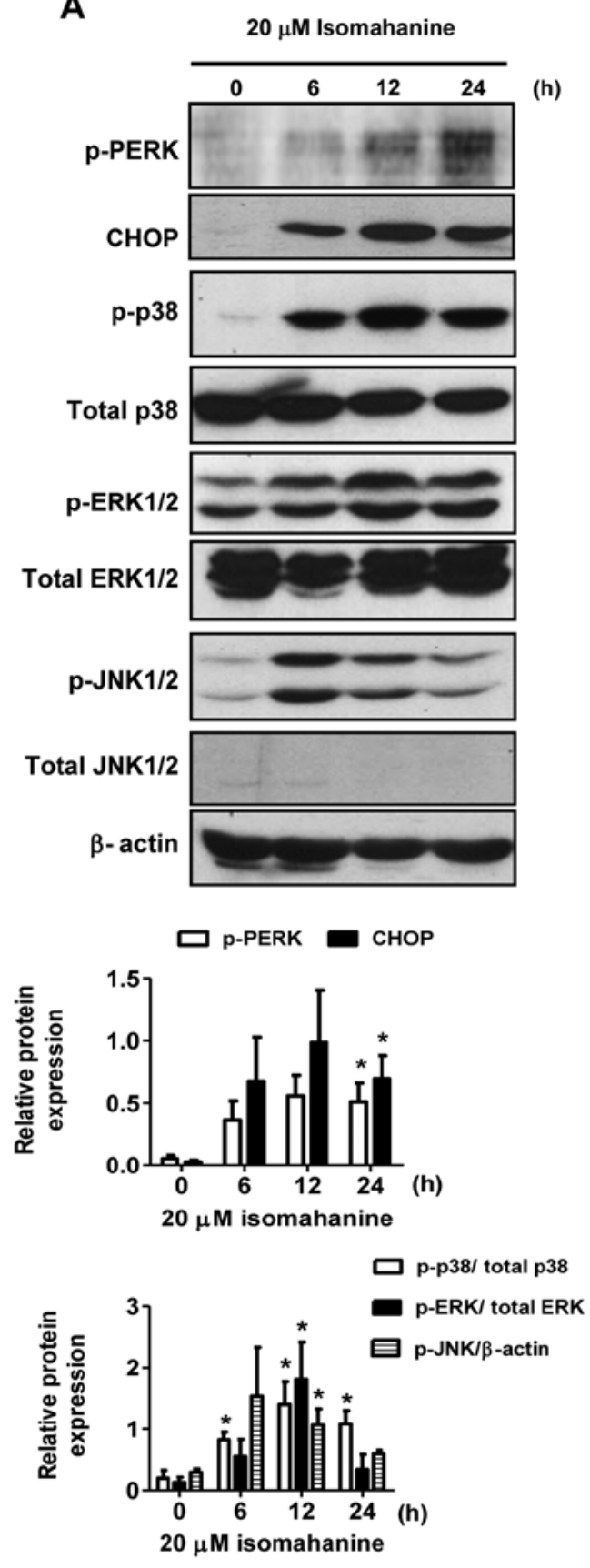

B
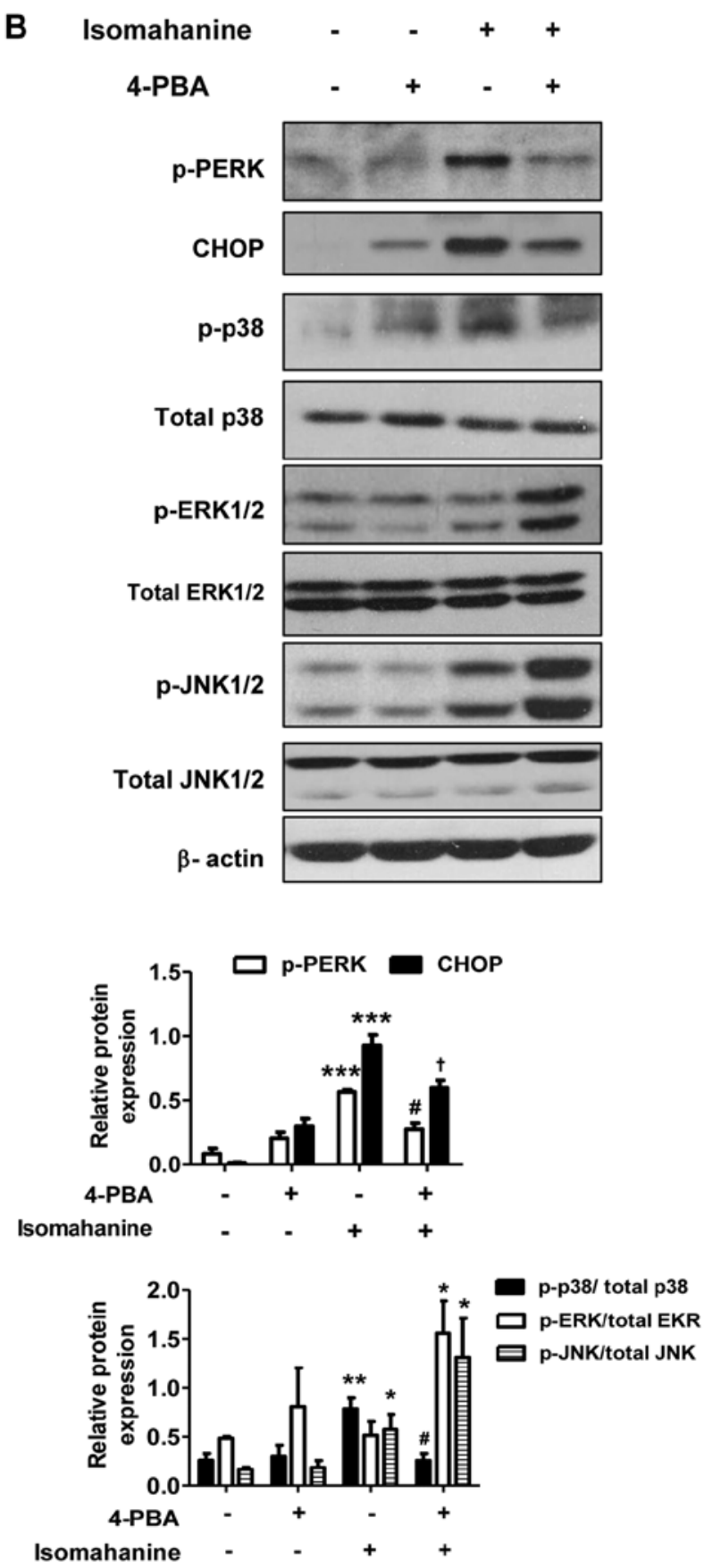

Figure 5. Isomahanine activates ER stress signaling and the MAPK signaling pathway in CLS-354/DX cells. (A) The cells were treated with isomahanine $(20 \mu \mathrm{M})$ for $0-24 \mathrm{~h}$. (B) The cells were pre-treated with 4-PBA $(5 \mathrm{mM})$ for $1 \mathrm{~h}$ prior to isomahanine $(20 \mu \mathrm{M})$ treatment for $6 \mathrm{~h}$. After treatment, the expression levels of ER stress markers (p-PERK and CHOP) and the expression levels of MAPKs (p38, ERK1/2 and JNK1/2) were determined by western blotting (upper images). The relative expression levels of the proteins were determined by densitometric analysis (lower images). Data are expressed as the mean \pm SEM of 3-4 independent experiments; ${ }^{*} \mathrm{p}<0.05,{ }^{* *} \mathrm{p}<0.01,{ }^{* * *} \mathrm{p}<0.001$ vs. $0 \mathrm{~h}$ or control; ${ }^{\dagger} \mathrm{p}<0.05,{ }^{\#} \mathrm{p}<0.01$ vs. the isomahanine treatment alone.

p38 MAPK (SB203580), ERK (U0126) and JNK (SP600125). As shown in Fig. 6A, SB203580 and U0126 significantly suppressed isomahanine-induced cell death, implying that p38 MAPK and ERK participate in cell death. The function of p38 MAPK andERK in the induction of apoptosis and autophagy was further examined using inhibitors SB203580 and U0126. Isomahanine activated caspase-3, LC3B-II and CHOP and concomitantly decreased the expression level of p62 as usual. SB203580 inhibited MAPKAPK-2, a downstream effector of p38 MAPK (Fig. 6B). The blockade of p38 MAPK completely inhibited isomahanine-activated caspase-3, LC3B-II and p62, but SB203580 did not affect CHOP expression (Fig. 6C). U0126 markedly inhibited ERK phosphorylation (Fig. 6B), and this attenuated only isomahanine-activated caspase-3 (Fig. 6C). These data indicated that p38 MAPK was an important cytotoxic mediator mediating both apoptosis and autophagic flux in response to isomahanine. Meanwhile, the activation of ERK, in part, regulated apoptosis under the tested conditions. As CHOP was not affected by MAPK inhibition, ER stress may be an upstream regulator involved in this activation. The overall cytotoxic mechanism of isomahanine against CLS-354/DX cells is illustrated in Fig. 6D. 
A

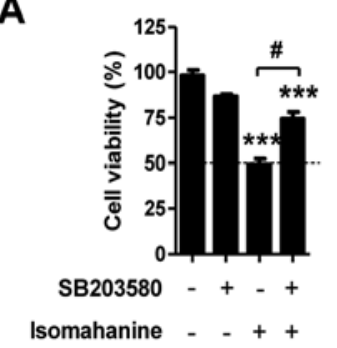

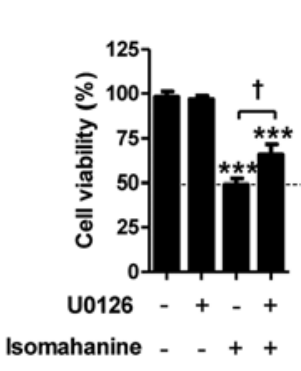

C
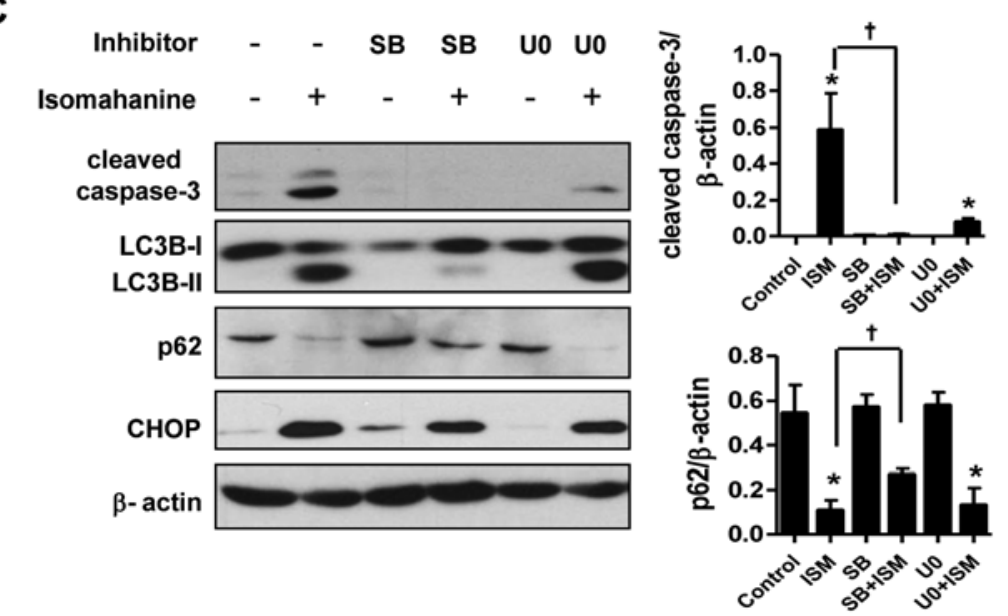

D

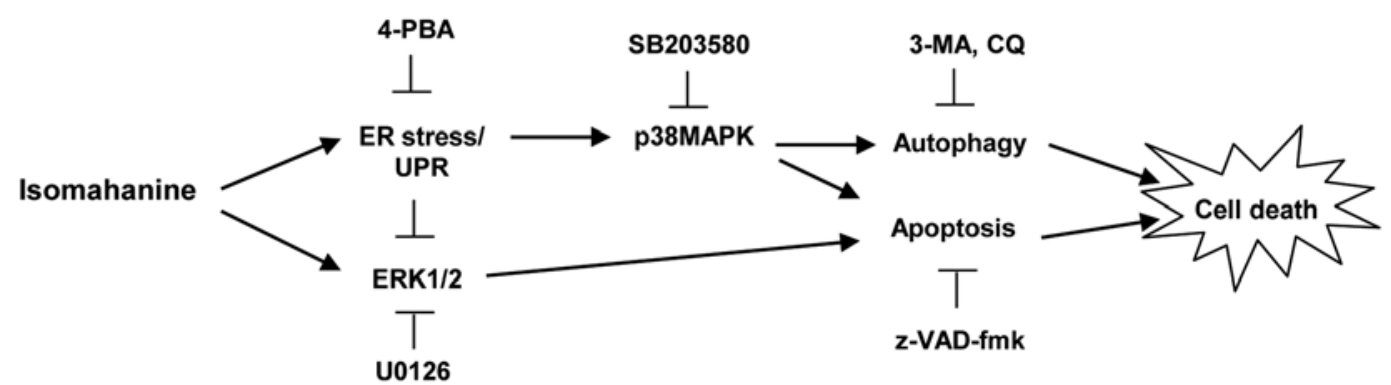

Figure 6. p38 MAPK mediates autophagy- and apoptosis-induced cell death in isomahanine-treated CLS-354/DX cells. (A) Cell viability of CLS-354/DX cells was assessed by MTT assay after treatment with isomahanine $(20 \mu \mathrm{M})$ in the absence or presence of inhibitors against p38 (20 $\mu \mathrm{M}$ SB203580) (left), ERK (10 $\mu \mathrm{M}$ U0126), and JNK (25 $\mu \mathrm{M}$ SP600125) for $24 \mathrm{~h}$. Data are expressed as mean \pm SEM of 4 independent experiments; ${ }^{* * *} \mathrm{p}<0.001 \mathrm{vs}$. the control without any treatment; ${ }^{\dagger} \mathrm{p}<0.05,{ }^{\sharp} \mathrm{p}<0.001 \mathrm{vs}$. the isomahanine treatment alone. Cells were treated with isomahanine (ISM) (20 $\left.\mu \mathrm{M}\right)$ for $24 \mathrm{~h}$ in the absence or presence of SB203580 (SB) $(20 \mu \mathrm{M})$ and U0126 (U0) $(10 \mu \mathrm{M})$. After treatment, (B) expression of MAPKs (MAPKAPK2, p38 and ERK1/2) was determined by western blotting, and (C) expression of apoptosis, autophagy, and ER stress markers was determined by western blot (left) and densitometric analyses of relative expression level (right). Data are expressed as mean \pm SEM of 3 independent experiments; ${ }^{*} \mathrm{p}<0.05,{ }^{* *} \mathrm{p}<0.01,{ }^{* * * *} \mathrm{p}<0.001$ vs. the control without any treatment; ${ }^{\dagger} \mathrm{p}<0.05$ vs. isomahanine treatment alone. (D) The proposed cytotoxic mechanism of isomahanine in CLS-354/DX cells. 3-MA, 3-methyladenine; 4-PBA, 4-phenylbutyric acid; CQ, chloroquine; UPR, unfolded protein response.

\section{Discussion}

MDR in advanced OSCC is associated with disease recurrence and poor patient survival $(30,31)$. To overcome multidrug-resistant cancer, identification of effective therapeutic regimens suitable for drug resistance in different tumors by targeting different cell-death pathways, particularly apoptosis and autophagy, has recently received attention $(5-8,32)$. Herein, we isolated a carbazole alkaloid, namely, isomahanine from $M$. koenigii leaves. The cytotoxic activity of this compound against the multidrug-resistant OSCC cell line CLS-354/DX was examined. We discovered that isomahanine $(20 \mu \mathrm{M})$ exerted cytotoxicity against CLS-354/DX cells regardless of resistance after stopping treatment. However, some cells were able to recover their proliferation following treatment with low concentrations of isomahanine $(10 \mu \mathrm{M})$. These cells may acquire resistant phenotypes due to a pro-survival autophagy; hence, the confirmation process may take a period of time from several months to a year (33). Isomahanine cytotoxicity was due to the induction of apoptosis and autophagic cell death in CLS-354/DX cells. This provided the notion that isomahanine may be useful for eliminating multidrug-resistant cancer cells.

CLS-354/DX cells spontaneously developed MDR through increased efflux activities of MRP and P-gp, increased expression of the MRP1 protein and elevated $\mathrm{IC}_{50}$ and $\mathrm{RI}$ values to cisplatin and camptothecin in comparison to the parental CLS-354/WT cells (4). Overexpression of MRP1 indicates intrinsic drug resistance, which confers resistance to a wide 
range of anticancer drugs, including camptothecin (34) and cisplatin (35). High expression of MRP1 was observed in tongue carcinoma, which was consequently associated with cisplatin resistance, suggesting the existence of this protein in head and neck cancer samples (36). A slightly increased MDR1 activity observed in CLS-354/DX cells may be due to MRP1, which has a significantly overlapping resistance profile with P-gp (37).

Isomahanine triggered CLS-354/DX cells to undergo apoptosis via the caspase-dependent pathway. Our observation was consistent with an earlier study that reported ROS-mediated caspase-dependent apoptosis in leukemia cells (21). Disruption of mitochondrial function may be a contributing effect to apoptosis. The release of cytochrome $c$ into the cytosol can lead to formation of apoptosomes and activation of caspase-3 through a mitochondrial-dependent pathway $(38,39)$. Next, we clearly demonstrated that isomahanine activated autophagic flux, which contributed to the cytotoxicity against CLS-354/DX cells. Accumulating data have shown that plant alkaloids selectively targeted autophagic cell death against multidrug-resistant cancer cells via different mechanisms, such as activation of AMP-activated protein kinase (40), ER stress, inhibition of the Akt/mTOR pathway (41) and induction of autophagic flux (42). Although the role of autophagy in cancer is still controversial as autophagy can either drive cell survival or cell death, pro-death is likely effective in particular multidrug-resistant cancer cells $(40,42)$. Hence, the isomahanine-induced autophagic pathway may circumvent conventional drug resistance in cancer.

In our experimental setting, ER stress was activated following isomahanine treatment as shown by the increased levels of p-PERK and CHOP. ER stress is a crucial stress signal caused by increased accumulation of unfolded proteins within the ER. From a structural point of view, isomahanine may disrupt protein folding in the ER, similar to the effect of plant alkaloid ellipticine-induced ER stress (43). Planar structure of the carbazole moiety and lipophilic property of isomahanine may contribute to ER disruption. Excessive or prolonged ER stress response can further initiate cell death pathways (15). Three canonical MAPKs (ERK1/2, JNK and p38) are recognized to be activated in response to ER stress-induced cell death (16). We found that $\mathrm{p} 38$ MAPK and ERK1/2 are involved in isomahanine-induced apoptosis. During ER stress, inositolrequiring protein-1 activates apoptosis signal-regulating kinase 1 , which is essential for the activation of p38 MAPK and JNK $(16,44)$. Isomahanine-induced p38 MAPK not only triggered apoptosis but also regulated autophagic flux. This may be due to the fact that autophagy requires p38 MAPK at the sequestration step during autophagosome formation induced by nutrient deprivation (45). This mechanism is consistent with previous studies on human gingival fibroblasts and human tongue squamous cell carcinoma cells $(45,46)$. p38 MAPK can act as an important mediator of apoptotic and autophagic cell death induced by isomahanine. ER stress induced or decreased ERK1/2 activation with different kinetics. Short-term induction of ER stress $(<6 \mathrm{~h})$ resulted in a decrease in ERK phosphorylation, while long-lasting activation $(>10 \mathrm{~h})$ induced ERK phosphorylation in hepatocellular carcinoma cells (47). In addition, we demonstrated that the MEK inhibitor rescued isomahanine-induced cell death and decreased cleavage of caspase-3. ERK-dependent apoptosis can be activated by mitochondrial cytochrome $c$ release or caspase- 8 activation, permanent cell cycle arrest, or autophagic vacuolization, depending on the different cellular contexts (48).

In conclusion, the present study elucidated the cytotoxic mechanism involved in the apoptosis and autophagy induced by isomahanine in multidrug-resistant OSCC cells. Isomahanine was capable of inducing ER stress, which may regulate apoptosis as well as autophagic cell death via p38 MAPK. The simultaneous autophagic cell death with apoptosis induced by isomahanine may provide a novel anticancer strategy to circumvent MDR in cancer.

\section{Acknowledgements}

The present study was supported by Strategic Scholarships Fellowships Frontier Research Networks for the Ph.D. Sandwich Program Doctoral Degree from the Office of the Higher Education Commission (OHEC), Thailand (06/2556), Walailak University (WU56113 and WU59201), Walailak University Fund for Graduate Studentship (27/2556 and WU55603), the Thailand Research Fund (DBG5980003), and Centre of Excellence for Innovation in Chemistry, OHEC. S.C. was partially supported by Structural and Computational Biology Research Group, Special Task Force for Activating Research (STAR), Faculty of Science, Rachadaphiseksomphot Endowment Fund, Chulalongkorn University, the Thailand Research Fund (TRF) (TRG5880222 and IRG 5780008), and the Institute for the Promotion of Teaching Science and Technology (IPST) under the Research Fund for DPST Graduate with First Placement (07/2557). We would like to thank Enago (https://www.enago.com/] for English language editing and reviewing of this manuscript.

\section{References}

1. Krishna Rao SV, Mejia G, Roberts-Thomson K and Logan R: Epidemiology of oral cancer in Asia in the past decade - an update (2000-2012). Asian Pac J Cancer Prev 14: 5567-5577, 2013.

2. Ferlay J, Soerjomataram I, Ervik M, Dikshit R, Eser S, Mathers C, Rebelo M, Parkin DM, Forman D and Bray F: GLOBOCAN 2012 v1.0, Cancer Incidence and Mortality Worldwide: IARC CancerBase No. 11 (Internet). International Agency for Research on Cancer, Lyon, France, 2014. doi:10.1002/ijc.29210 PMID:25220842. Accessed October, 9, 2014.

3. Wang C, Liu XQ, Hou JS, Wang JN and Huang HZ: Molecular mechanisms of chemoresistance in oral cancer. Chin J Dent Res 19: 25-33, 2016.

4. Wu Q, Yang Z, Nie Y, Shi Y and Fan D: Multi-drug resistance in cancer chemotherapeutics: Mechanisms and lab approaches. Cancer Lett 347: 159-166, 2014.

5. Meschini S, Condello M, Marra M, Formisano G, Federici E and Arancia G: Autophagy-mediated chemosensitizing effect of the plant alkaloid voacamine on multidrug resistant cells. Toxicol In Vitro 21: 197-203, 2007.

6. Chow MJ, Licona C, Pastorin G, Mellitzer G, Ang WH and Gaiddon C: Structural tuning of organoruthenium compounds allows oxidative switch to control ER stress pathways and bypass multidrug resistance. Chem Sci 7: 4117-4124, 2016.

7. Kumar P,Zhang DM, Degenhardt K and Chen ZS: Autophagy and transporter-based multi-drug resistance. Cells 1: 558-575, 2012.

8. Xuan Y and Hu X: Naturally-occurring shikonin analogues - a class of necroptotic inducers that circumvent cancer drug resistance. Cancer Lett 274: 233-242, 2009.

9. Gewies A: Introduction to apoptosis. ApoReview: 1-26, 2003.

10. Millimouno FM, Dong J, Yang L, Li J and Li X: Targeting apoptosis pathways in cancer and perspectives with natural compounds from mother nature. Cancer Prev Res 7: 1081-1107, 2014. 
11. Klionsky DJ and Emr SD: Autophagy as a regulated pathway of cellular degradation. Science 290: 1717-1721, 2000.

12. Zhang XJ, Chen S, Huang KX and Le WD: Why should autophagic flux be assessed? Acta Pharmacol Sin 34: 595-599, 2013.

13. Chen N and Karantza V: Autophagy as a therapeutic target in cancer. Cancer Biol Ther 11: 157-168, 2011.

14. Jiao JW and Wen F: Tanshinone IIA acts via p38 MAPK to induce apoptosis and the down-regulation of ERCC1 and lung-resistance protein in cisplatin-resistant ovarian cancer cells. Oncol Rep 25: 781-788, 2011.

15. Xu C, Bailly-Maitre B and Reed JC: Endoplasmic reticulum stress: Cell life and death decisions. J Clin Invest 115: 2656-2664, 2005.

16. Darling NJ and Cook SJ: The role of MAPK signalling pathways in the response to endoplasmic reticulum stress. Biochim Biophys Acta 1843: 2150-2163, 2014.

17. Szegezdi E, Logue SE, Gorman AM and Samali A: Mediators of endoplasmic reticulum stress-induced apoptosis. EMBO Rep 7: 880-885, 2006.

18. Wang Y, Wang JW, Xiao X, Shan Y, Xue B, Jiang G, He Q, Chen J, $\mathrm{Xu} \mathrm{HG}, \mathrm{Zhao} \mathrm{RX}$, et al: Piperlongumine induces autophagy by targeting p38 signaling. Cell Death Dis 4: e824, 2013.

19. Ma K, Zhang C, Huang MY, Li WY and Hu GQ: Cinobufagin induces autophagy-mediated cell death in human osteosarcoma U2OS cells through the ROS/JNK/p38 signaling pathway. Oncol Rep 36: 90-98, 2016.

20. Li JP, Yang YX, Liu QL, Pan ST, He ZX, Zhang X, Yang T, Chen XW, Wang D, Qiu JX, et al: The investigational Aurora kinase A inhibitor alisertib (MLN8237) induces cell cycle $\mathrm{G}_{2} / \mathrm{M}$ arrest, apoptosis, and autophagy via p38 MAPK and $\mathrm{Akt} / \mathrm{mTOR}$ signaling pathways in human breast cancer cells Drug Des Devel Ther 9: 1627-1652, 2015.

21. Roy MK, Thalang VN, Trakoontivakorn G and Nakahara K Mechanism of mahanine-induced apoptosis in human leukemia cells (HL-60). Biochem Pharmacol 67: 41-51, 2004.

22. Ito C, Itoigawa M, Nakao K, Murata T, Tsuboi M, Kaneda N and Furukawa $\mathrm{H}$ : Induction of apoptosis by carbazole alkaloids isolated from Murraya koenigii. Phytomedicine 13: 359-365, 2006.

23. Sarkar S, Dutta D, Samanta SK, Bhattacharya K, Pal BC, Li J, Datta K, Mandal C and Mandal C: Oxidative inhibition of Hsp90 disrupts the super-chaperone complex and attenuates pancreatic adenocarcinoma in vitro and in vivo. Int J Cancer 132: 695-706, 2013.

24. Shaikh MS, Karpoormath R, Thapliyal N, Rane RA, Palkar MB, Faya AM, Patel HM, Alwan WS, Jain K and Hampannavar GA: Current perspective of natural alkaloid carbazole and its derivatives as antitumor agents. Anticancer Agents Med Chem 15: 1049-1065, 2015.

25. Das R, Bhattacharya K, Sarkar S, Samanta SK, Pal BC and Mandal C: Mahanine synergistically enhances cytotoxicity of 5-fluorouracil through ROS-mediated activation of PTEN and p53/p73 in colon carcinoma. Apoptosis 19: 149-164, 2014

26. Das R, Bhattacharya K, Samanta SK, Pal BC and Mandal C: Improved chemosensitivity in cervical cancer to cisplatin: Synergistic activity of mahanine through STAT3 inhibition. Cancer Lett 351: 81-90, 2014

27. Chen CM, Syu JP, Way TD, Huang LJ, Kuo SC, Lin CT and Lin CL: BC3EE2,9B, a synthetic carbazole derivative, upregulates autophagy and synergistically sensitizes human GBM8901 glioblastoma cells to temozolomide. Int J Mol Med 36: 1244-1252, 2015.

28. Reisch J, Goj O, Wickramasinghe A, Herath B and Henkel G: Carbazole alkaloids from seeds of Murraya koenigii. Phytochemistry 31: 2877-2879, 1992.

29. Chamulitrat W, Schmidt R, Chunglok W, Kohl A and Tomakidi P: Epithelium and fibroblast-like phenotypes derived from HPV16 E6/E7-immortalized human gingival keratinocytes following chronic ethanol treatment. Eur J Cell Biol 82: 313-322, 2003

30. Kim JW, Park Y, Roh JL, Cho KJ, Choi SH, Nam SY and Kim SY: Prognostic value of glucosylceramide synthase and $\mathrm{P}$-glycoprotein expression in oral cavity cancer. Int J Clin Oncol 21: 883-889, 2016.
31. Friedrich RE, Punke C and Reymann A: Expression of multi-drug resistance genes ( $m d r l, m r p l, b c r p)$ in primary oral squamous cell carcinoma. In Vivo 18: 133-147, 2004.

32. Saraswathy $M$ and Gong S: Different strategies to overcome multidrug resistance in cancer. Biotechnol Adv 31: 1397-1407, 2013.

33. Harada K, Ferdous T and Ueyama Y: Establishment of 5-fluorouracil-resistant oral squamous cell carcinoma cell lines with epithelial to mesenchymal transition changes. Int J Oncol 44: 1302-1308, 2014.

34. Yajima T, Ochiai H, Uchiyama T, Takano N, Shibahara T and Azuma T: Resistance to cytotoxic chemotherapy-induced apoptosis in side population cells of human oral squamous cell carcinoma cell line Ho-1-N-1. Int J Oncol 35: 273-280, 2009.

35. Negoro K, Yamano Y, Fushimi K, Saito K, Nakatani K, Shiiba M, Yokoe H, Bukawa H, Uzawa K, Wada T, et al: Establishment and characterization of a cisplatin-resistant cell line, KB-R, derived from oral carcinoma cell line, KB. Int J Oncol 30: 1325-1332, 2007.

36. Zhang B, Liu M, Tang HK, Ma HB, Wang C, Chen X and Huang HZ: The expression and significance of MRP1, LRP, TOPOII $\beta$, and BCL2 in tongue squamous cell carcinoma. J Oral Pathol Med 41: 141-148, 2012.

37. Kathawala RJ, Gupta P, Ashby CR Jr and Chen ZS: The modulation of $\mathrm{ABC}$ transporter-mediated multidrug resistance in cancer: A review of the past decade. Drug Resist Updat 18: 1-17, 2015.

38. Roy MK, Thalang VN, Trakoontivakorn G and Nakahara K: Mahanine, a carbazole alkaloid from Micromelum minutum, inhibits cell growth and induces apoptosis in U937 cells through a mitochondrial dependent pathway. Br J Pharmacol 145: 145-155, 2005.

39. Sinha S, Pal BC, Jagadeesh S, Banerjee PP, Bandyopadhaya A and Bhattacharya S: Mahanine inhibits growth and induces apoptosis in prostate cancer cells through the deactivation of Akt and activation of caspases. Prostate 66: 1257-1265, 2006.

40. Law BY, Mok SW, Chan WK, Xu SW, Wu AG, Yao XJ, Wang JR, Liu L and Wong VK: Hernandezine, a novel AMPK activator induces autophagic cell death in drug-resistant cancers. Oncotarget 7: 8090-8104, 2016

41. Hasanain M, Bhattacharjee A, Pandey P, Ashraf R, Singh N Sharma S, Vishwakarma AL, Datta D, Mitra K and Sarkar J: $\alpha$-Solanine induces ROS-mediated autophagy through activation of endoplasmic reticulum stress and inhibition of Akt/mTOR pathway. Cell Death Dis 6: e1860, 2015.

42. Meschini S, Condello M, Calcabrini A, Marra M, Formisano G, Lista P, De Milito A, Federici E and Arancia G: The plant alkaloid voacamine induces apoptosis-independent autophagic cell death on both sensitive and multidrug resistant human osteosarcoma cells. Autophagy 4: 1020-1033, 2008.

43. Hägg M, Berndtsson M, Mandic A, Zhou R, Shoshan MC and Linder S: Induction of endoplasmic reticulum stress by ellipticine plant alkaloids. Mol Cancer Ther 3: 489-497, 2004.

44. Tobiume K, Matsuzawa A, Takahashi T, Nishitoh H, Morita K, Takeda K, Minowa O, Miyazono K, Noda T and Ichijo H: ASK1 is required for sustained activations of JNK/p38 MAP kinases and apoptosis. EMBO Rep 2: 222-228, 2001.

45. Kim DS, Kim JH, Lee GH, Kim HT, Lim JM, Chae SW, Chae HJ and Kim HR: p38 Mitogen-activated protein kinase is involved in endoplasmic reticulum stress-induced cell death and autophagy in human gingival fibroblasts. Biol Pharm Bull 33: 545-549, 2010.

46. Pan ST, Qin Y, Zhou ZW, He ZX, Zhang X, Yang T, Yang YX, Wang D, Qiu JX and Zhou SF: Plumbagin induces $\mathrm{G}_{2} / \mathrm{M}$ arrest, apoptosis, and autophagy via p38 MAPK- and PI3K/Akt/ mTOR-mediated pathways in human tongue squamous cell carcinoma cells. Drug Des Devel Ther 9: 1601-1626, 2015

47. Dai R, Chen R and Li H: Cross-talk between PI3K/Akt and MEK/ERK pathways mediates endoplasmic reticulum stress-induced cell cycle progression and cell death in human hepatocellular carcinoma cells. Int J Oncol 34: 1749-1757, 2009.

48. Cagnol S and Chambard JC: ERK and cell death: Mechanisms of ERK-induced cell death - apoptosis, autophagy and senescence. FEBS J 277: 2-21, 2010 